EPJ Web of Conferences 47, 13003 (2013)

DOI: $10.1051 /$ epjconf/20134713003

(C) Owned by the authors, published by EDP Sciences, 2013

\title{
A spectral differential characterization of low-mass companions
}

\author{
N. Kostogryz',2,a, M. Kürster ${ }^{2}$, T. Yakobchuk ${ }^{1}$, Y. Lyubchik ${ }^{1}$ and M. Kuznetsov ${ }^{1}$ \\ ${ }^{1}$ Main Astronomical Observatory of NAS of Ukraine, 27 Akademika Zabolotnoho St., \\ 03680 Kyiv, Ukraine \\ ${ }^{2}$ Max-Planck-Institute for Astronomy, Königstuhl 17, 69117 Heidelberg, Germany
}

\begin{abstract}
We present a new approach with which the dynamical mass of low-mass companions around cool stars can be found. In order to discover companions to late-type stars the stellar spectrum is removed. For this we substract two spectra obtained at different orbital phases from each other in order to discover the companion spectrum in the difference spectrum in which the companion lines appear twice (positive and negative signal). The resulting radial velocity difference of these two signals provides the true mass of the companion. For our test case GJ1046, an M2V dwarf with a low-mass companion that most likely is a brown dwarf we select the CO line region in the K-band. We show that the dynamical mass of a faint companion to an $\mathrm{M}$ dwarf can be determined using our spectral differential technique. Only if the companion rotates rapidly and has a small radial velocity amplitude due to a high mass, does blending occur for all lines so that our approach fails. In addition to determining the companion mass, we restore the single companion spectrum from the difference spectrum using singular value decomposition.
\end{abstract}

\section{INTRODUCTION}

In this paper we introduce a spectral differential method to the suite of techniques with which the dynamical mass and hence the true nature of low-mass companions can be determined. We develop our technique around the example of GJ1046b, which is a companion to the inactive old M2.5 V star that was found by $[2,6]$. This object has a minimum mass of 27 Jupiter mass $\left(\mathrm{M}_{\mathrm{Jup}}\right)$ that was derived from RV measurements, and from a combination of the RV measurements with Hipparcos astrometry a maximum mass of $112 \mathrm{M}_{\text {Jup }}$ was found by [2]. With a separation of $0.42 \mathrm{AU}$ the companion is close to its host star and consequently it is located in the brown dwarf desert.

Around solar-type stars, few candidates are located in the brown dwarf desert all of which have large star-to-companion brightness ratios. A more favourable brightness contrast can be found in systems comprised of a brown dwarf orbiting an M-type star in the brown dwarf desert. There are several known candidates for probable brown dwarf companions to M-type stars, such as GJ1046 ([2]), GJ595, GJ623, and GJ84 ([4]). For this paper we chose GJ1046 as a sample to develop our technique, but certainly, we can apply this method to other brown dwarf companions to M-type stars.

\section{METHOD OF DIFFERENCE SPECTRUM}

Our method aims at discovering companions to late-type stars by removing the stellar spectrum through subtraction of spectra obtained at different orbital phases and identifying the companion spectrum in the difference spectrum. Using near-infrared spectra of sufficient resolution one can attempt to search for absorption lines of the companion in the spectrum. In our approach we take one spectrum each near the

\footnotetext{
ae-mail: kosn@mao.kiev.ua
}

This is an Open Access article distributed under the terms of the Creative Commons Attribution License 2.0, which permits unrestricted use, distribution, and reproduction in any medium, provided the original work is properly cited. 


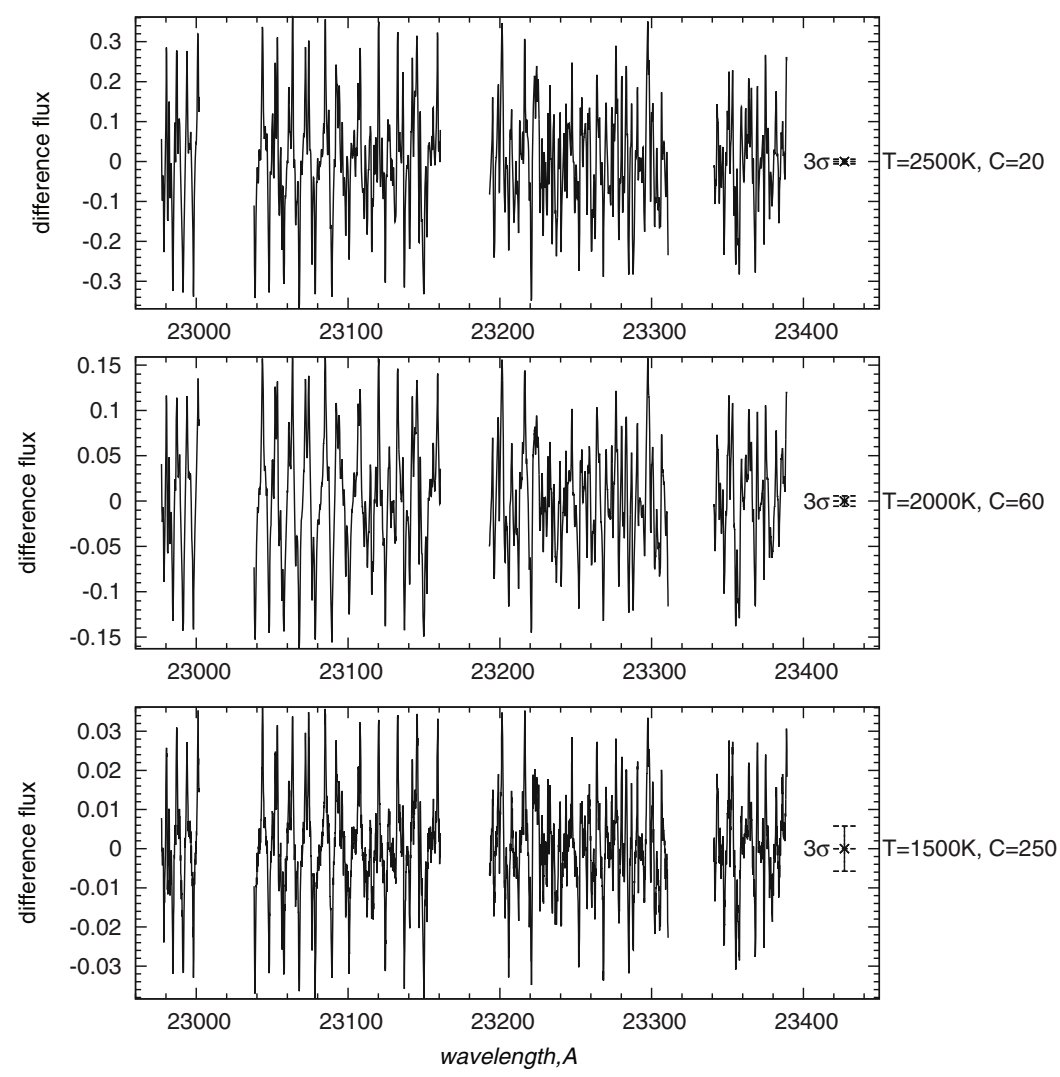

Figure 1. Simulated difference spectra obtained from observations at companion's RV maximum and minimum. The stellar spectrum cancels. Three panels for effective temperatures $2500 \mathrm{~K}, 2000 \mathrm{~K}$ and $1500 \mathrm{~K}$, and contrasts $C=20,60,250$, respectively, for the minimum possible companion mass $m_{2, \min }=26.85 M_{J u p}$. The solid line is the companion difference flux with photon noise added, the crosses together with the 3 -sigma error bars depict just the photon noise.

maximum and the minimum of the known radial velocity curve of the star, shift them in wavelength in such a way that the stellar line systems co-align and subtract them from each other thereby removing the stellar signal (the photon noise still has to be dealt with). What one is left with is a spectrum that consists of the difference of two spectra of the companion, but taken at different radial velocities. The companion lines will therefore appear twice in this difference spectrum, once as a positive signal and once as a negative signal. By determining the radial velocity difference of these two signals one can obtain the star-to-companion mass ratio and, if the stellar mass is known, one can determine the true mass of the companion and thereby its nature.

The spectrum of the invisible companion will be detectable when the star-to-companion brightness ratio (hereafter called "the contrast") of the two components of the system is sufficiently small. The best wavelength range for low-mass star observations is the near-infrared (NIR). [3] provide the absolute magnitude - spectral type dependence in the $\mathrm{J}$ and $\mathrm{K}$ bands for objects from $\mathrm{L} 0$ to $\mathrm{T} 9$, and it can be seen that in the $\mathrm{J}$ band the contrast is less favourable for these spectral types than in the $\mathrm{K}$ band. Therefore, we selected the $\mathrm{K}$ band, where sharp CO lines are found. To realize our approach we need an NIR spectrograph with high resolution of the order 50,000 - 100,000 such as the cryogenic high-resolution infrared spectrograph CRIRES that is mounted at the Very Large Telescope of the European Southern Observatory. 


\section{Hot Planets and Cool Stars}

Table 1. Determined companion masses for different rotational velocities of the companion: $5 \mathrm{kms}^{-1}, 10 \mathrm{kms}^{-1}$, $15 \mathrm{kms}^{-1}$ and $20 \mathrm{kms}^{-1}$. Input $M_{\text {in }}$ were calculated from the difference spectra.

\begin{tabular}{|c|c|c|c|c|}
\hline$v$ & $M_{\text {in }}$ & & $M_{\text {out }}\left(M_{\text {Jup }}\right)$ & \\
\hline $\sin i$ & $\left(M_{\text {Jup }}\right)$ & $2500 \mathrm{~K}$ & $2000 \mathrm{~K}$ & $1500 \mathrm{~K}$ \\
\hline 5 & 26.85 & $26.11 \pm 0.22$ & $26.93 \pm 0.32$ & $27.08 \pm 0.14$ \\
10 & 26.85 & $27.00 \pm 0.28$ & $26.60 \pm 0.49$ & $26.65 \pm 0.27$ \\
15 & 26.85 & $26.80 \pm 0.35$ & $26.45 \pm 0.58$ & $26.85 \pm 0.41$ \\
20 & 26.85 & $26.91 \pm 0.58$ & $26.67 \pm 0.47$ & $26.76 \pm 0.24$ \\
\hline 5 & 111.74 & $107.7 \pm 1.3$ & $107.0 \pm 4.0$ & $110.0 \pm 1.9$ \\
10 & 86.91 & $85.7 \pm 1.8$ & $83.9 \pm 1.3$ & $88.0 \pm 2.5$ \\
15 & 67.04 & $64.49 \pm 0.89$ & $65.98 \pm 0.60$ & $71.3 \pm 1.9$ \\
20 & 54.57 & $54.5 \pm 1.4$ & $56.2 \pm 2.4$ & $56.1 \pm 1.1$ \\
\hline
\end{tabular}

The absence of high-resolution spectroscopic observational data for GJ1046 leads us to simulate CRIRES observations in order to investigate the feasibility for the detection of the companion in the difference spectrum. For this purpose we take the synthetic spectra described in [1]. We generate Poisson distributed photon noise with a signal-to-noise ratio (SNR) equal to 500 per spectral pixel for each of the spectra from the two different observational epochs.

\section{RESULTS}

By planning for observations of GJ1046 near the minimum and maximum of its RV curve and then subtracting the obtained spectra from each other we aim at the discovery of the CO lines of the brown dwarf candidate companion to the M2.5V host star. Figure 1 presents simulations of difference spectra taken at maximum amd minimum stellar RV, previously shifted to correct for stellar orbital and Earth's barycentric motions thereby making the stellar spectrum cancel. In [1] several kinds of uncertainties concerning misalignment of the two spectra during subtraction, imperfect orbital solution of the primary star and activity of the host star are considered.

Taking a signal-to-noise ratio of 500, we determine the difference spectrum for the three different effective temperatures of the companion $2500 \mathrm{~K}, 2000 \mathrm{~K}, 1500 \mathrm{~K}$ (Fig. 1 shows this for the minimum companion mass but the result for maximum mass is very similar). As is seen from Figure 1, the companion signal is much stronger than the photon noise even for the object with an effective temperature of $1500 \mathrm{~K}$. Therefore, we can confirm that it is feasible to detect this difference companion spectrum in the planned observations with CRIRES.

Once we have obtained the difference spectrum, we will try to find the mass of the companion. To this end it is important to consider the broadening of the spectral lines due to rotation of the companion. The faster the rotation of the companion, the broader the $\mathrm{CO}$ lines will be and therefore more blended lines will appear in the difference spectrum. All our synthetic spectra were convolved with a rotational profile corresponding to projected rotational velocities of $5 \mathrm{kms}^{-1}, 10 \mathrm{kms}^{-1}, 15 \mathrm{kms}^{-1}$ and $20 \mathrm{kms}^{-1}$ to study how rotation of the companion influences the determination of the mass.

Table 1 shows the determined RV shifts and masses from the difference companion spectrum with various rotational velocities. For a companion with the minimum possible mass no blending effect occurs in the difference spectra which results in a correct mass determination (Table 1) for all adopted rotational velocities. But, for the maximum companion mass it is only possible for rotational velocities of $5 \mathrm{kms}^{-1}$, to find the correct wavelength shift and therefore the mass of the companion (all obtained masses are within $\sim 3 \sigma$ of the true mass). The lower part of Table 1 provides in the second column the maximum mass values for which the correct mass determination is still possible. For higher rotational velocities, the obtained masses are wrong because of blending of the CO-lines in the difference 


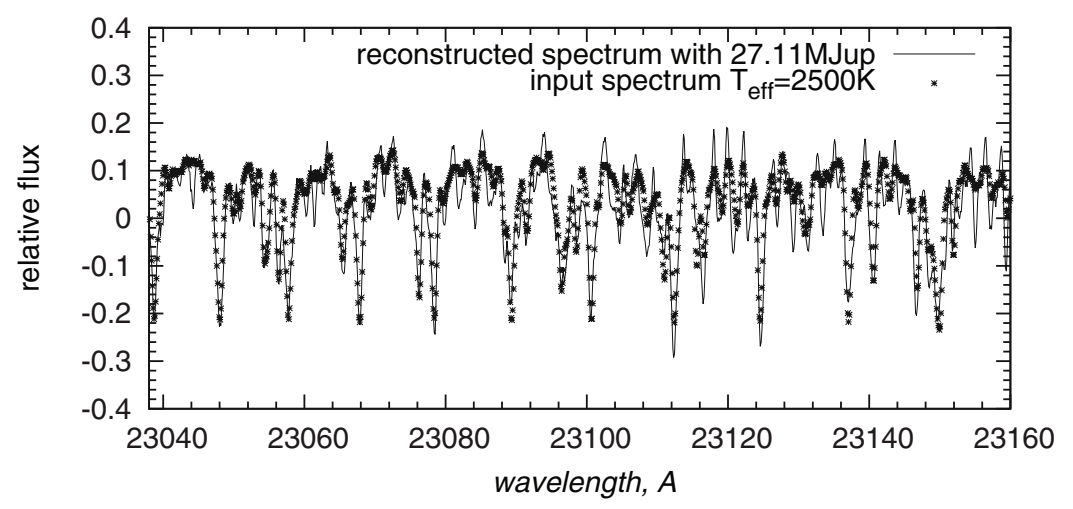

Figure 2. Companion spectrum (solid line) reconstructed from difference spectrum for a companion with a mass of 27.11 $M_{\text {Jup }}$. The crosses show the input spectrum of the companion with the same signal-to-noise ratio.

spectrum. To reconstruct the (single) companion spectrum from the difference spectrum, we apply the method of singular value decomposition using the algorithm by [5].

The reconstructed companion spectrum is shown in Fig. 2. Note that for display purposes we show it only for the wavelength region covered by the second CRIRES detector in the wavelength setting selected by us. We reconstructed this companion spectrum for the case of a companion mass of $27.11 M_{\text {Jup }}$, effective temperature of $2500 \mathrm{~K}$, and rotational velocity of $5 \mathrm{kms}^{-1}$ (cf. Table 1 ). As is seen from Fig. 2, the main features are reconstructed, but there are discrepancies in the finer details.

\section{CONCLUSIONS}

In this paper, we have presented a spectral differential technique for the detection and characterization of an invisible close companion to late-type stars. We show that companion difference spectra with effective temperatures of $2500 \mathrm{~K}, 2000 \mathrm{~K}$ and $1500 \mathrm{~K}$ would be possible to detect with CRIRES for companions to an $\mathrm{M} 2.5 \mathrm{~V}$ star. We demonstrate that the determination of the companion mass can be made with very high accuracy for slowly rotating companions. Faster rotation of the companion complicates its mass determination, especially for high companion masses. Knowing the true wavelength shift value we have reconstructed the spectrum using singular value decomposition.

\section{References}

[1] Kostogryz, N., Kürster, M., Yakobchuk, T., Lyubchik, Y., Kuznetsov, M., submitted to Astronomische Nachrichten (2012)

[2] Kürster, M., Endl, M., Reffert, S., A\&A 483, 869 (2008)

[3] Knapp G.R., Leggett S.K., Fan X., Marley M.S., Geballe T.R., et al., AJ 127, 3553 (2004)

[4] Nidever D.L., Marcy G.W., Butler R.P., et al., ApJS 141, 503N (2002)

[5] Press W.H., Teukolsky S.A., Vetterling W.T., Flannery B.P., 1992, Numerical recipes in C. The art of scientific computing (Cambridge: University Press)

[6] Zechmeister M., Kürster M., Endl M., A\&A 505, 859 (2009) 